\title{
Semi-Markovian Model of Monotonous System Maintenance with Regard to its Elements' Deactivation and Age
}

\author{
Yuriy E. Obzherin, Aleksey I. Peschansky \\ Sevastopol National Technical University, Sevastopol, Ukraine \\ E-mail:vmsevntu@mail.ru \\ Received June 18, 2010; revised July 26, 2010; accepted August 1, 2010
}

\begin{abstract}
An explicit form of reliability and economical stationary performance indexes for monotonous multicomponent system with regard to its elements' maintenance has been found. The maintenance strategy investigated supposes preventive maintenance execution for elements that has attained certain operating time to failure. Herewith for the time period of elements' maintenance or restoration operable elements, functionally connected with the failed ones, are deactivated. The problems of maintenance execution frequency optimization have been solved. For the model building the theory of semi-Markovian processes with a common phase field of states is used.
\end{abstract}

Keywords: Maintenance, Semi-Markovian Process, System Stationary Characteristics, System Performance Indexes Optimization

\section{Introduction}

One of the methods of the complex technical systems' reliability improvement is their maintenance. The review of the results concerning this subject can be found in the works [1-3]. One of the strategies of a single-component system maintenance is the strategy known in literature as "Depending-on-age restoration" [4-6]. This strategy being used, the system is considered to be completely restored after its failure. If the system has been operating without failures for the given time period $\tau$, then its maintenance, after which it is completely restored, is executed. In [7] semi-Markovian model of the abovementioned strategy for multicomponent monotonous system maintenance under assumption that any system's element failure does not result in deactivation of elements that are in up state, are functionally connected with the failed ones, and do not belong to any up-state path has been built.

The goal of the present article is to build semi-Markovian model of maintenance in age of a multicomponent system's elements with regard to their deactivation. On the basis of the model built it is necessary to define stationary reliability and economical performance indexes of the system and to solve the problem of elements' main- tenance optimal terms determination.

\section{The Problem Definition and Mathematical Model Building}

Let us consider $\mathrm{N}$-component system with a monotonous structure and describe the strategy of its elements' maintenance. At the time zero $t=0$ system operation begins and an acceptable operating time to failure level (age) $\tau_{i}$ for each $i$-element of system is determined. On attaining this level element's planned maintenance is carried out. The failure-free operation time of system's $i$ element is a random value (RV) $\alpha_{i}$ with distribution function (DF) $F_{i}(t)$. Unless system's $i$-element fails by the moment $\tau_{i}$, element's planned maintenance that restores it completely begins. The maintenance lasts random period of time $\beta_{i}^{p}$ with DF $G_{i}^{p}(t)$.

If system's $i$-element has failed by the moment $\tau_{i}$, its failure is discovered instantly and its emergency restoration (ER) begins. This restoration lasts RV $\beta_{i}$ with DF $G_{i}(t)$. As a result of ER, an element is restored completely and the whole maintenance process occurs again.

Let us assume that due to emergency failure or to the beginning of some element's maintenance the operable 
elements that do not belong to any other up-state path are deactivated. Besides, the elements in state of ER or maintenance, the restoration of which would not result in any up-state path formation, are deactivated.

The elements deactivated have the same operable level at the moment of their activation. The latter happens at the end of element's ER or maintenance under the condition of simultaneous up-state path formation.

Time diagram of system operation is shown in Figure 1.

Let us begin semi-Markovian (SM) model building of the system. To begin with the phase field of states should be defined. Each element of system can be in three physical states:

1 - in up state or deactivated in up state;

0 - in state of restoration or deactivated in state of restoration;

2 - in state of maintenance or deactivated in state of maintenance.

System's physical states will be indicated with a set of vectors $D=\left\{\bar{d}=\left(d_{1}, \ldots, d_{N}\right), d_{k}=0,1,2 ; k=\overline{1, N}\right\}$. The component $d_{k}$ of vector $\bar{d}$ denotes the physical state of system's $k$-element.

The physical states to exhibit SM property, they should be extended. With this purpose we will indicate the number of element that was last to change its state. Let us add continuous components, denoting time periods of elements' dwelling in their states. In the code of extended state these time periods will be indicated by vector $\bar{x}^{(i)}=\left(x_{1}, \ldots, x_{i-1}, 0, x_{i+1}, \ldots, x_{N}\right)$.

Besides, in accordance with the chosen maintenance strategy we will introduce vector $\bar{u}=\left(u_{1}, \ldots, u_{N}\right)$, the components of which indicate elements' operating time since the last restoration of their up state, to the code of system's states.

Thus, the system's phase field of SM states with re- gard to its elements' maintenance execution is the following:

$$
E^{*}=\{i \bar{d} \bar{x}(i) \bar{u}, \quad i=\overline{1, N}\}
$$

The significance of the code of states:

$i$ is the number of element that was last to change its physical state;

$d_{k}=0,1,2$ is the code of system's $k$-element physical state;

$x_{k}$ is time period between $i$-element's last state change and the nearest moment of $k$-element's change $\left(x_{i}=0\right)$ regardless of deactivation time; and if $d_{k}$ $=1$ then $x_{k}$ is the time period till the nearest emergency failure of $k$-element;

$u_{k}$ is operating time to failure of $k$-element since the end of its last ER or maintenance. If $d_{k}=2$ it is considered that $u_{k}=\tau_{k}$. At the moment of $i$-element's transition to up state after its maintenance or ER its operating time is equal zero: $u_{i}=0$.

Let us indicate $I_{d}$ a set of numbers of elements deactivated in the state $i \bar{d} x \bar{u}(i) \bar{u}, i=\overline{1, N}$. System dwelling time periods are defined by ratios:

$$
\theta_{i \bar{d} \bar{x} \bar{x}^{(i)} \bar{u}}=\gamma_{i}^{\left(d_{i}\right)} \wedge \Lambda_{\substack{k \neq i \\ k \notin I_{d}}} x_{k} \Lambda_{\substack{k \in \Omega_{d}^{1} \\ k \notin I_{d}}}\left(\tau_{k}-u_{k}\right)
$$

where $\Lambda$ is a sign of minimum; $\Omega_{d}^{1}$ is a set of numbers of vector $\bar{d}$ components that are equal to 1 ,

$$
\gamma_{i}^{\left(d_{i}\right)}=\left\{\begin{array}{l}
\alpha_{i}, d_{i}=1, \\
\beta_{i}, d_{i}=0, \\
\beta_{i}{ }^{p}, d_{i}=2 .
\end{array}\right.
$$

Let us describe the probabilities (probability densities) of embedded Markovian chain (EMC) $\left\{\xi_{n}, n \geq 0\right\}$ transition. It is necessary to note that $i$-element can change its physical state 1 into the state 0 (ER) and into the

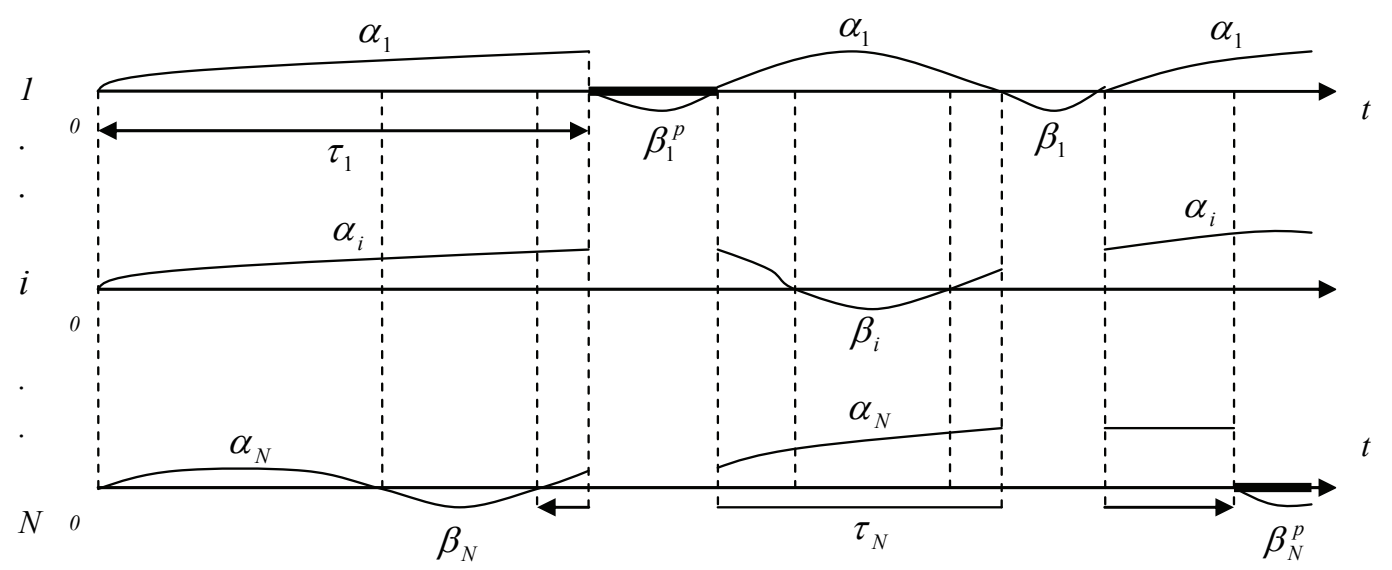

Figure 1. Time diagram of the system operation with elements' deactivation after the first element failure and with regard to their maintenance in age. 
state 2 (maintenance) but the states 0 and 2 can be changed only into the state 1 .

Let us indicate

$$
z_{i, I_{d}}=\bigwedge_{\substack{k \neq i \\ k \notin I_{d}}} x_{k} \wedge \Lambda_{\substack{k \in \Omega_{d}^{1} \\ k \notin I_{d}}}\left(\tau_{k}-u_{k}\right)
$$

and let $\Omega_{d}^{0}, \Omega_{d}^{2}$ be sets of numbers of vector $\bar{d}$ components that are equal to 0 and 2 respectively.

The state $i \bar{d} x \bar{u}, i=\overline{1, N}$ admits the following transitions:

1) to the set of states $i \overline{d^{\prime}}-(i) \overline{u^{\prime}}, d_{i}^{\prime} \neq 2$ with the probability density of transition

$p_{i \bar{d} x^{(i)} \bar{u}}^{i \overline{d^{\prime} \bar{x}^{(i)}}-\overline{u^{\prime}}}=\psi_{i}^{\left(d_{i}\right)}\left(z_{i, I_{d}}-y\right)$, where $y<z_{i, I_{d}}, \psi_{i}^{\left(d_{i}\right)}(\cdot)$, is the density of probability distribution of RV $\gamma_{i}^{\left(d_{i}\right)}, d_{k}^{\prime}=d_{k}$, $k \neq i ; \quad x_{k}^{\prime}=x_{k}-\left(z_{i, I_{d}}-y\right), \quad k \neq i, k \notin I_{d} ; x_{k}^{\prime}=x_{k}$, $k \in I_{d}$;

$$
\begin{aligned}
& u_{k}^{\prime}=\left\{\begin{array}{cc}
u_{k}+z_{i, I_{d}}-y, & k \in \Omega_{d}^{1}, k \notin I_{d}, \\
u_{k}, & k \in \Omega_{d}^{0}, k \in I_{d}, \quad k \neq i, \\
\tau_{k}, & k \in \Omega_{d}^{2},
\end{array}\right. \\
& u_{i}^{\prime}=\left\{\begin{array}{cc}
u_{i}+z_{i, I_{d}}-y, & i \in \Omega_{d}^{1}, \\
0, & i \in \Omega_{d}^{0} \cup \Omega_{d}^{2} ;
\end{array}\right.
\end{aligned}
$$

2) to the set of states $i \overline{d^{\prime}} \overline{x^{\prime}} \bar{u}^{(i)}, \quad d_{i}=1, d_{i}^{\prime}=2$ with transition probability $P_{i \bar{d} x^{-(i)} \bar{u}}^{i \overline{d^{\prime} \bar{x}^{(i)}}-\overline{u^{\prime}}}=\bar{F}_{i}\left(\tau_{i}\right)$, where $d_{k}^{\prime}=d_{k}$,

$$
\begin{gathered}
k \neq i ; \quad x_{k}^{\prime}=x_{k}-\tau_{i}, \quad k \neq i, \quad k \notin I_{d} ; x_{k}^{\prime}=x_{k}, k \in I_{d} ; \\
u_{k}^{\prime}=\left\{\begin{array}{cc}
u_{k}+\tau_{i}, & k \in \Omega_{d}^{1}, k \notin I_{d}, \\
u_{k}, & k \in \Omega_{d}^{0}, k \in I_{d}, \\
\tau_{k}, & k \in \Omega_{d}^{2} ;
\end{array}\right.
\end{gathered}
$$

$3)$ to the set of states $j \overline{d^{\prime}} \overline{x^{\prime}}(j) \overline{u^{\prime}}, \quad j \neq i, j \notin I_{d} \quad$ with the probability density of transition

$$
p_{i \bar{d} \bar{x}^{(i)} \bar{u}}^{j \bar{d}^{-x^{(j)}} \overline{u^{\prime}}}=\psi_{i}^{\left(d_{i}\right)}\left(z_{i, I_{d}}+y\right) \text {, where } y>0, d_{k}^{\prime}=d_{k}, k \neq j \text {, }
$$$$
x_{i}^{\prime}=y, \quad x_{k}^{\prime}=x_{k}-z_{i, I_{d}}, \quad k \neq i, j \text {, }
$$

$$
\begin{aligned}
& u_{j}^{\prime}=\left\{\begin{array}{c}
u_{j}+z_{i, I_{d}}, j \in \Omega_{d}^{1}, d_{j}^{\prime}=0, \\
\tau_{j}, \quad j \in \Omega_{d}^{1}, d_{j}^{\prime}=2, \\
0, \quad j \in \Omega_{d}^{0} \cup \Omega_{d}^{2},
\end{array}\right. \\
& u_{k}^{\prime}=\left\{\begin{array}{cc}
u_{k}+z_{i, I_{d}}, & k \in \Omega_{d}^{1}, k \notin I_{d}, \\
u_{k}, & k \in \Omega_{d}^{0}, k \in I_{d}, \\
\tau_{k}, & k \in \Omega_{d}^{2},
\end{array}\right.
\end{aligned}
$$

Let us assume that the conditions of stationary distribution $\rho(\cdot) \quad[8,9]$ existence and uniqueness for EMC $\left\{\xi_{n}, n \geq 0\right\}$ are fulfilled. The following theorem takes place.

Theorem. The stationary distribution of EMC $\left\{\xi_{n}, n \geq 0\right\}$ is defined by the following expressions:

$$
\begin{aligned}
& \rho(i \bar{d} \bar{x}(i) \bar{u})=\left\{\begin{array}{l}
\rho \prod_{k \in \Omega_{d}^{0}} f_{k}\left(u_{k}\right) \bar{G}_{k}\left(x_{k}\right) \prod_{k \in \Omega_{d}^{1}} f_{k}\left(u_{k}+x_{k}\right) \prod_{k \in \Omega_{d}^{2}} \bar{F}_{k}\left(\tau_{k}\right) \bar{G}_{k}^{p}\left(x_{k}\right), i \notin \Omega_{d}^{1}, x_{i}=0, \\
\rho \prod_{k \in \Omega_{d}^{0}} f_{k}\left(u_{k}\right) \bar{G}_{k}\left(x_{k}\right) \prod_{\substack{k \in \Omega_{d}^{1} \\
k \neq i}} f_{k}\left(u_{k}+x_{k}\right) \prod_{k \in \Omega_{d}^{2}} \bar{F}_{k}\left(\tau_{k}\right) \bar{G}_{k}^{p}\left(x_{k}\right), i \in \Omega_{d}^{1},
\end{array}\right. \\
& \rho=\left[\sum_{d \in D^{*}}\left[\sum_{\substack{i \in \Omega_{d}^{1} \\
i \notin I_{d} \\
i \neq i}} \prod_{\substack{k \neq 1 \\
k \neq i}}^{N} T_{k}^{\left(d_{k}\right)}\left(\tau_{k}\right)+\sum_{\substack{i \in \Omega_{d}^{0} \\
i \notin I_{d}}} F_{i}\left(\tau_{i}\right) \prod_{\substack{k=1 \\
k \neq i}}^{N} T_{k}^{\left(d_{k}\right)}\left(\tau_{k}\right)+\sum_{\substack{i \in \Omega_{d}^{2} \\
i \notin I_{d}}} \bar{F}_{i}\left(\tau_{i}\right) \prod_{\substack{k=1 \\
k \neq i}}^{N} T_{k}^{\left(d_{k}\right)}\left(\tau_{k}\right)\right]\right]^{-1} \\
& T_{k}^{(1)}\left(\tau_{k}\right)=\int_{0}^{\tau_{k}} \bar{F}_{k}(t) d t, \quad T_{k}^{(0)}\left(\tau_{k}\right)=F_{k}\left(\tau_{k}\right) M \beta_{k}, \quad T_{k}^{(0)}\left(\tau_{k}\right)=\bar{F}_{k}\left(\tau_{k}\right) M \beta_{k}^{p} .
\end{aligned}
$$

Theorem proving. The stationary distribution of probabilities $\rho(B)$ obeys the system of integral equations [8]

$$
\rho(B)=\int_{E^{*}} \rho(d z) P(z, B) .
$$

For example, the equation of this system for the state $i \bar{d} x \bar{u}, \quad d_{i}=0, i=\overline{1, N} ; i \notin I_{d}$; is as follows:

$$
\begin{aligned}
& \rho(i \bar{d} \bar{x}(i) \bar{u})=f_{m}\left(x_{m}+u_{m, I}\right) \rho\left(m \overline{d^{\prime} \bar{x}^{\prime \prime}}(m) \overline{u^{\prime \prime}}\right) \\
& +\sum_{\substack{j \in \Omega_{d^{\prime}}^{0} \cup \Omega_{d^{\prime}}^{2} \\
j \notin I_{d^{\prime}}}} \int_{0}^{u_{m, I}} \psi_{j}^{\left(d_{j}^{\prime}\right)}\left(t+x_{j}\right) \rho\left(j \overline{d^{\prime}}\left(\overline{x^{\prime}}(j) \overline{u^{\prime}}\right) d t,\right.
\end{aligned}
$$


$d_{i}^{\prime}=1, d_{k}^{\prime}=d_{k}, k \neq i, u_{m, I}=\Lambda_{\substack{k \in \Omega_{d^{\prime}}^{\prime} \\ k \notin I_{d^{\prime}}}} u_{k}$

By the direct substitution one can check that Formula
(2) define the solution of this equation. For the state $i \bar{d} \bar{x} \bar{u}$ we deal with $d_{i}=0, d_{i}^{\prime}=1, \Omega_{d^{\prime}}^{1}-\{i\}=\Omega_{d}^{1}$, $\Omega_{d^{\prime}}^{0} \cup\{i\}=\Omega_{d}^{0}, \Omega_{d^{\prime}}^{2}=\Omega_{d}^{2}$. Substituting (2) to the second member of Equation (3) we get the following results:

$$
\begin{aligned}
& f_{m}\left(u_{m, I}+x_{m}\right) \prod_{k \in \Omega_{d^{\prime}}^{0}} f_{k}\left(u_{k}\right) \bar{G}_{k}\left(x_{k}+u_{m, I}\right) \prod_{\substack{k \in \Omega_{d d^{\prime}}^{1} \\
k \neq m}} f_{k}\left(u_{k}+x_{k}\right) \prod_{k \in \Omega_{d^{\prime}}^{2}} \bar{F}_{k}\left(\tau_{k}\right) \bar{G}_{k}^{p}\left(x_{k}+u_{m, I}\right)+ \\
& {\left[\sum_{\substack{j \in \Omega_{d^{\prime}}^{0} \\
j \notin I_{d^{\prime}}}} \int_{0}^{u_{m}, I} g_{j}\left(x_{j}+t\right) f_{j}\left(u_{j}\right) \prod_{\substack{k \in \Omega_{\Omega^{\prime}}^{0} \\
k \notin I_{d^{\prime}}, k \neq j}} f_{k}\left(u_{k}\right) \bar{G}_{k}\left(x_{k}+t\right) \prod_{\substack{k \in \Omega_{d^{\prime}}^{1} \\
d^{\prime}}} f_{k}\left(u_{k}+x_{k}\right) \prod_{\substack{k \in \Omega_{d^{\prime}}^{2} \\
k \notin I_{d^{\prime}}}} \bar{F}_{k}\left(\tau_{k}\right) \bar{G}_{k}^{p}\left(x_{k}+t\right) d t+\right.} \\
& \left.\sum_{\substack{j \in \Omega^{2} \\
j \notin I_{d^{\prime}}}}^{u_{m, I}} \int_{0}^{u_{d^{\prime}}} g_{j}^{p}\left(x_{j}+t\right) \prod_{\substack{k \in \Omega_{d d^{\prime}}^{0} \\
k \notin I_{d^{\prime}}}} f_{k}\left(u_{k}\right) \bar{G}_{k}\left(x_{k}+t\right) \prod_{\substack{k \in \Omega_{d^{\prime}}^{1} \\
d^{\prime}}} f_{k}\left(u_{k}+x_{k}\right) \bar{F}_{j}\left(\tau_{j}\right) \prod_{\substack{k \in \Omega_{d^{\prime}}^{2} \\
k \notin I_{d^{\prime}}, k \neq j}} \bar{F}_{k}\left(\tau_{k}\right) \bar{G}_{k}^{p}\left(x_{k}+t\right) d t\right] \times \\
& \prod_{\substack{k \in \Omega_{d^{\prime}}^{0} \\
k \in I_{d^{\prime}}}} f_{k}\left(u_{k}\right) \bar{G}_{k}\left(x_{k}\right) \prod_{\substack{k \in \Omega_{d^{\prime}} \\
k \in I_{d^{\prime}}}} \bar{F}_{k}\left(\tau_{k}\right) \bar{G}_{k}^{p}\left(x_{k}\right)= \\
& \prod_{k \in \Omega_{d^{\prime}}^{0}} f_{k}\left(u_{k}\right) \bar{G}_{k}\left(x_{k}+u_{m, I}\right) \prod_{k \in \Omega_{d^{\prime}}^{1}} f_{k}\left(u_{k}+x_{k}\right) \prod_{k \in \Omega_{d^{\prime}}^{2}} \bar{F}_{k}\left(\tau_{k}\right) \bar{G}_{k}^{p}\left(x_{k}+u_{m, I}\right)- \\
& \prod_{k \in \Omega_{d^{\prime}}^{1}} f_{k}\left(u_{k}+x_{k}\right) \prod_{\substack{k \in \Omega_{d^{\prime}}^{0} \\
k \in I_{d^{\prime}}}} f_{k}\left(u_{k}\right) \bar{G}_{k}\left(x_{k}\right) \prod_{\substack{k \in \Omega_{d^{\prime}}^{2} \\
k \in I_{d^{\prime}}}} \bar{F}_{k}\left(\tau_{k}\right) \bar{G}_{k}^{p}\left(x_{k}\right) \times \\
& \int_{0}^{u_{m, I} I} \frac{\partial}{\partial t}\left[\prod_{\substack{k \in \Omega_{d^{\prime}}^{0} \\
k \notin I_{d^{\prime}}}} f_{k}\left(u_{k}\right) \bar{G}_{k}\left(x_{k}+t\right) \prod_{\substack{k \in \Omega_{d^{\prime}}^{2} \\
k \notin I_{d^{\prime}}}} \bar{F}_{k}\left(\tau_{k}\right) \bar{G}_{k}^{p}\left(x_{k}+t\right)\right] d t= \\
& \prod_{k \in \Omega_{d^{\prime}}^{1}} f_{k}\left(u_{k}+x_{k}\right) \prod_{k \in \Omega_{d^{\prime}}^{0}} f_{k}\left(u_{k}\right) \bar{G}_{k}\left(x_{k}\right) \prod_{k \in \Omega_{d^{\prime}}^{2}} \bar{F}_{k}\left(\tau_{k}\right) \bar{G}_{k}^{p}\left(x_{k}\right)= \\
& f_{i}\left(u_{i}\right) \prod_{k \in \Omega_{d}^{1}} f_{k}\left(u_{k}+x_{k}\right) \prod_{\substack{k \in \Omega_{d}^{0} \\
k \neq i}} f_{k}\left(u_{k}\right) \bar{G}_{k}\left(x_{k}\right) \prod_{k \in \Omega_{d}^{2}} \bar{F}_{k}\left(\tau_{k}\right) \bar{G}_{k}^{p}\left(x_{k}\right)=\frac{1}{\rho} \rho\left(i \bar{d} \bar{x}^{(i)} u\right) .
\end{aligned}
$$

In the same way it can be checked that Formula (2) define the stationary distributions for the rest of system's states. The constant $\rho$ is determined due to normalization condition.

\section{Definition of System Stationary Characteristics}

Let us define the following system stationary performance indexes: mean stationary operating time to failure $T_{+}^{*}\left(\tau_{1}, \ldots, \tau_{N}\right) ;$ mean stationary restoration time
$T_{-}^{*}\left(\tau_{1}, \ldots, \tau_{N}\right)$; stationary steady state availability factor (SSAF) $K_{u}^{*}\left(\tau_{1}, \ldots, \tau_{N}\right) ;$ mean specific income $S^{*}\left(\tau_{1}, \ldots, \tau_{N}\right)$ per calendar time unit, and mean specific expenses $C^{*}\left(\tau_{1}, \ldots, \tau_{N}\right)$ per time unit of system's good state.

Let us divide the phase field $E^{*}$ of system's states into two non-overlapping subsets $E_{+}^{*}$ and $E_{-}^{*} ; E_{+}^{*}$ is a subset of up states, $E_{-}^{*}$ is a subset of down states:

$$
\begin{aligned}
& E_{+}^{*}=\left\{i \bar{d}^{-(i)} \bar{u}, \bar{d} \in D_{+}^{*}, \quad i=\overline{1, N}\right\} \\
& E_{-}^{*}=\left\{i \bar{d}^{-(i)} \bar{u}, \bar{d} \in D_{-}^{*}, \quad i=\overline{1, N}\right\}
\end{aligned}
$$


Here $D_{+}^{*}\left(D_{-}^{*}\right)$ is a set of vectors $\bar{d}$ the components of which are equal to the codes of physical states of system's elements; this system is in a subset of up (down) states $E_{+}^{*}\left(E_{-}^{*}\right)$.

Mean stationary operating time to failure $T_{+}^{*}$, mean stationary restoration time $T_{-}^{*}$, and stationary SSAF $K_{u}^{*}$ of the system will be estimated with the help of formulas $[8,9]$

$$
\begin{aligned}
& T_{+}^{*}=\frac{\int_{E_{+}^{*}} m(z) \rho(d z)}{\int_{E_{-}^{*}} \rho(d z) P\left(z, E_{+}^{*}\right)}, T_{-}^{*}=\frac{\int_{E_{-}^{*}} m(z) \rho(d z)}{\int_{E_{-}^{*}} \rho(d z) P\left(z, E_{+}^{*}\right)}, \\
& K_{u}^{*}=\frac{T_{+}^{*}}{T_{+}^{*}+T_{-}^{*}} \\
& \int_{E_{+}^{*}} m(z) \rho(d z)=\sum_{d \in D_{+}^{*}} \sum_{i=1}^{N} \int_{U} d \overline{\hat{u}} \int_{R_{+}^{N, i}} \rho(i \bar{d} \bar{x} \bar{x} \bar{u}) d \bar{x} \bar{x}_{0}^{(i)} \int_{0}^{z_{i, I} I_{d}} \bar{\Psi}_{i}^{\left(d_{i}\right)}(t) d t=
\end{aligned}
$$

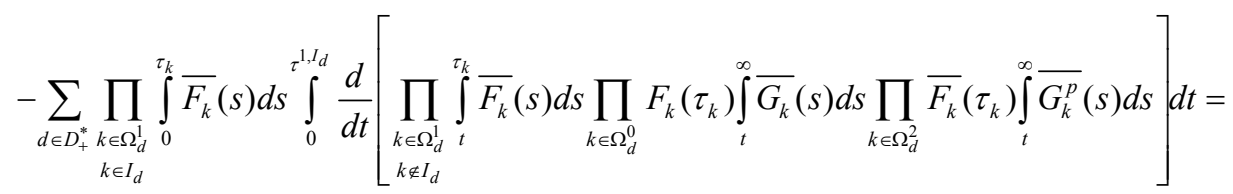

$$
\begin{aligned}
& \sum_{d \in D_{+}^{*}} \prod_{k \in \Omega_{d}^{1}} \int_{0}^{\tau_{k}} \overline{F_{k}}(s) d s \prod_{k \in \Omega_{d}^{0}} M \beta_{k} F_{k}\left(\tau_{k}\right) \prod_{k \in \Omega_{d}^{2}} M \beta_{k}^{p} \overline{F_{k}}\left(\tau_{k}\right)=\sum_{d \in D_{+}^{*}} \prod_{k=1}^{N} T_{k}^{\left(d_{k}\right)}\left(\tau_{k}\right) .
\end{aligned}
$$

where $\rho(\cdot)$ is the stationary distribution of EMC $\left\{\xi_{n}, n \geq 0\right\}, m(z)$ are mean time periods of system's dwelling in its states, $P\left(z, E_{+}^{*}\right)$ are probabilities of EMC $\left\{\xi_{n}, n \geq 0\right\}$ transition from down to up states.

To define the stationary indexes with the help of Formula (4) it is necessary to define the basic characteristics included in these formulas.

Let us begin with the integral $\int_{E_{+}^{*}} m(z) \rho(d z)$. Mean time period of system's dwelling in the state $i \bar{d} \bar{x}^{(i)} \bar{u}$ is found by the formula $M\left[\theta_{\bar{i} \overline{\bar{x}^{(i)}} u}\right]=\int_{0}^{z_{i, I_{d}}} \bar{\Psi}_{i}^{\left(d_{i}\right)}(t) d t$, where

Here

$$
\tau^{1, I_{d}}=\Lambda_{\substack{k \in \Omega_{d}^{1} \\ k \notin I_{d}}} \tau_{k}, R_{+}^{N, i}=\left\{\bar{x}^{-(i)}, x_{k} \geq 0, k=\overline{1, N}\right\}, U=\left\{\overline{\hat{u}}=\left(u_{i_{1}}, \ldots u_{i_{s}}\right), 0 \leq u_{i_{r}} \leq \tau_{k}, i_{r}=k, k \in \Omega_{d}^{0}, \Omega_{d}^{1}\right\} .
$$

The values $T_{k}^{\left(d_{k}\right)}\left(\tau_{k}\right)$ have the following significance: $T_{k}^{(1)}\left(\tau_{k}\right)$ is mean time period of $k$-element dwelling in up state, and $T_{k}^{(0)}\left(\tau_{k}\right)+T_{k}^{(2)}\left(\tau_{k}\right)$ is mean time period of this element dwelling in down state during its regeneration.

Analogically, we have

$$
\int_{E_{-}^{*}} m(z) \rho(d z)=\sum_{d \in D_{-}^{*}} \prod_{k \in \Omega_{d}^{1}} \int_{0}^{\tau_{k}} \overline{F_{k}}(s) d s \prod_{k \in \Omega_{d}^{0}} M \beta_{k} F_{k}\left(\tau_{k}\right) \prod_{k \in \Omega_{d}^{2}} M \beta_{k}^{p} \overline{F_{k}}\left(\tau_{k}\right)=\sum_{d \in D_{-}^{*}} \prod_{k=1}^{N} T_{k}^{\left(d_{k}\right)}\left(\tau_{k}\right) .
$$

Let us calculate the integral in denominators of ratios (4). It is necessary to note that the transitions to $E_{+}^{*}$ can occur from the subset $E_{-}^{*^{\prime}} \subset E_{-}^{*}$ only with the probability equal to 1 where $E_{-}^{*^{\prime}}=\left\{i \bar{d} \bar{x}(i) \bar{u}, \bar{d} \in D_{-}^{*}, i \in \Omega_{d}^{0} \cup \Omega_{d}^{2}, i \notin I_{d}\right\}$. We have

$$
\int_{E_{-}^{*}} \rho(d z) P\left(z, E_{+}^{*}\right)=\int_{E_{-}^{*^{\prime}}} \rho(d z)=\sum_{d \in D_{-}^{*}}\left[\sum_{\substack{i \in \Omega_{d}^{0} \\ i \notin I_{d}}} F_{i}\left(\tau_{i}\right) \prod_{\substack{k=1 \\ k \neq i}}^{N} T_{k}^{\left(d_{k}\right)}\left(\tau_{k}\right)+\sum_{\substack{i \in \Omega_{d}^{2} \\ i \notin I_{d}}} \bar{F}_{i}\left(\tau_{i}\right) \prod_{\substack{k=1 \\ k \neq i}}^{N} T_{k}^{\left(d_{k}\right)}\left(\tau_{k}\right)\right]
$$


Thus, the Formula (4) are transformed into

$$
\begin{aligned}
& T_{+}^{*}\left(\tau_{1}, \ldots, \tau_{N}\right)=\frac{\sum_{d \in D_{+}^{*}} \prod_{k=1}^{N} T_{k}^{\left(d_{k}\right)}\left(\tau_{k}\right)}{\sum_{d \in D_{-}^{*}}\left[\sum_{\substack{i \in \Omega_{d}^{0} \\
i \notin I_{d}}} F_{i}\left(\tau_{i}\right) \prod_{\substack{k=1 \\
k \neq i}}^{N} T_{k}^{\left(d_{k}\right)}\left(\tau_{k}\right)+\sum_{\substack{i \in \Omega_{d}^{2} \\
i \notin I_{d}}} \bar{F}_{i}\left(\tau_{i}\right) \prod_{\substack{k=1 \\
k \neq i}}^{N} T_{k}^{\left(d_{k}\right)}\left(\tau_{k}\right)\right]}, \\
& T_{-}^{*}\left(\tau_{1}, \ldots, \tau_{N}\right)=\frac{\sum_{d \in D_{-}^{*}} \prod_{k=1}^{N} T_{k}^{\left(d_{k}\right)}\left(\tau_{k}\right)}{\sum_{d \in D_{-}^{*}}\left[\sum_{\substack{i \in \Omega_{d}^{0} \\
i \notin I_{d}}} F_{i}\left(\tau_{i}\right) \prod_{\substack{k=1 \\
k \neq i}}^{N} T_{k}^{\left(d_{k}\right)}\left(\tau_{k}\right)+\sum_{\substack{i \in \Omega_{d}^{2} \\
i \notin I_{d}}} \bar{F}_{i}\left(\tau_{i}\right) \prod_{\substack{k=1 \\
k \neq i}}^{N} T_{k}^{\left(d_{k}\right)}\left(\tau_{k}\right)\right]},
\end{aligned}
$$

$$
K_{u}^{*}\left(\tau_{1}, \ldots, \tau_{N}\right)=\frac{\sum_{d \in D_{+}^{*}} \prod_{k=1}^{N} T_{k}^{\left(d_{k}\right)}\left(\tau_{k}\right)}{\sum_{d \in D^{*}} \prod_{k=1}^{N} T_{k}^{\left(d_{k}\right)}\left(\tau_{k}\right)} .
$$

Let us determine system stationary characteristics $T_{+}^{*}, T_{-}^{*}, K_{u}^{*}\left(\tau_{1}, \ldots, \tau_{N}\right)$ by means of elements' SSAF $K_{i}\left(\tau_{i}\right)$ defined by the formulas $[4,5]$ :

$$
K_{i}\left(\tau_{i}\right)=\frac{T_{i}^{(1)}\left(\tau_{i}\right)}{T_{i}^{(1)}\left(\tau_{i}\right)+T_{i}^{(0)}\left(\tau_{i}\right)+T_{i}^{(2)}\left(\tau_{i}\right)}, i=\overline{1, N} .
$$

Let $M_{i}, i=\overline{1, \omega}$, be all the different sets of elements of system paths, and $\Phi_{i}, i=\overline{1, s}$ be sets of elements of system [4] sections; $A\left(\Phi_{i}\right)\left(A\left(M_{i}\right)\right)$ is a set of deactivated elements of section $\Phi_{i}$ (of $M_{i}$ path). One should pay attention that according to the definition the elements not belonging to the set of elements of path are in down state, i.e., are in a state 0 or 2 . The elements not belonging to the set of elements of section are in up state 1.

The Formulas' (5)-(7) transformation of averages products' sums lead to the following result:

$$
\begin{gathered}
T_{+}^{*}\left(\tau_{1}, \ldots, \tau_{N}\right)=\frac{\sum_{i=1}^{\infty} \prod_{n \in M_{i}} K_{n}\left(\tau_{n}\right) \prod_{\substack{n=1 \\
n \notin M_{i}}}^{N}\left(1-K_{n}\left(\tau_{n}\right)\right)}{\sum_{i=1}^{s} \sum_{\substack{j \in \Phi_{i} \\
j \notin A\left(\Phi_{i}\right)}} \frac{1}{T_{j}^{(0)}+T_{j}^{(2)}} \prod_{\substack{n=1 \\
n \notin \Phi_{i}}}^{N} K_{n}\left(\tau_{n}\right) \prod_{n \in \Phi_{i}}\left(1-K_{n}\left(\tau_{n}\right)\right)}, \\
T_{-}^{*}\left(\tau_{1}, \ldots, \tau_{N}\right)=\frac{\sum_{i=1}^{s} \prod_{n=1}^{N} K_{n}\left(\tau_{n}\right) \prod_{n \in \Phi_{i}}\left(1-K_{n}\left(\tau_{n}\right)\right)}{\sum_{i=1}^{s} \sum_{\substack{j \notin \Phi_{i} \\
j \notin A\left(\Phi_{i}\right)}} \frac{1}{T_{j}^{(0)}+T_{j}^{(2)}} \prod_{n=1}^{N} K_{n}\left(\tau_{n}\right) \prod_{n \in \Phi_{i}}\left(1-K_{n}\left(\tau_{n}\right)\right)}, \\
K_{u}\left(\tau_{1}, \ldots, \tau_{N}\right)=\frac{\sum_{i=1}^{\infty} \prod_{n \in M_{i}} K_{n}\left(\tau_{n}\right) \prod_{n=1}^{N}\left(1-K_{n}\left(\tau_{n}\right)\right)}{\sum_{i=1}^{\omega} \prod_{n \in M_{i}} K_{n}\left(\tau_{n}\right) \prod_{n=1}^{N}\left(1-K_{n}\left(\tau_{n}\right)\right)+\sum_{i=1}^{s} \prod_{n=1}^{N} K_{n}\left(\tau_{n}\right) \prod_{n \in \Phi_{i}}\left(1-K_{n}\left(\tau_{n}\right)\right)} .
\end{gathered}
$$

To define mean specific income $S\left(\tau_{1}, \ldots, \tau_{N}\right)$ per calendar time unit and mean specific expenses $C^{*}\left(\tau_{1}, \ldots, \tau_{N}\right)$ per time unit of system's up state the Formula [10] will be used

$$
S^{*}=\frac{\int_{E^{*}} m(z) f_{s}(z) \rho(d z)}{\int_{E^{*}} m(z) \rho(d z)}, C^{*}=\frac{\int_{E^{*}} m(z) f_{c}(z) \rho(d z)}{\int_{E_{+}^{*}} m(z) \rho(d z)}
$$


where $f_{s}(z), f_{c}(z)$ are functions defining income and expenses respectively in each state. These functions are as follows:

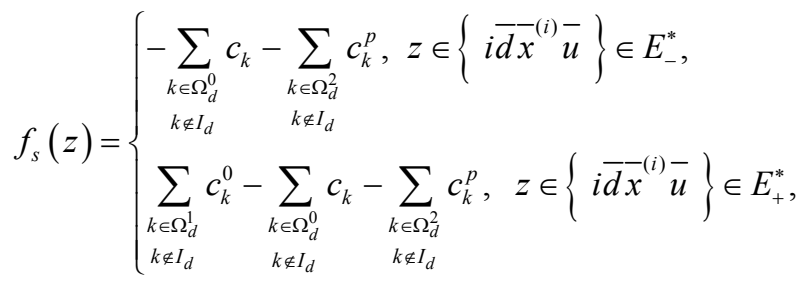

$$
f_{c}(z)=\sum_{\substack{k \in \Omega_{d}^{0} \\ k \notin I_{d}}} c_{k}+\sum_{\substack{k \in \Omega_{d}^{2} \\ k \notin I_{d}}} c_{k}^{p}, \quad z \in\{i \bar{d} \bar{x}(i) \bar{u}\} \in E^{*} .
$$

Here $c_{i}^{0}, c_{i}$ and $c_{i}^{p}, i=\overline{1, N}$, are income per time unit of system's up state, expenses per time unit of ER, and expenses per time unit of system's $i$-element maintenance respectively.

The Formula (11) can be transformed into the following expressions:

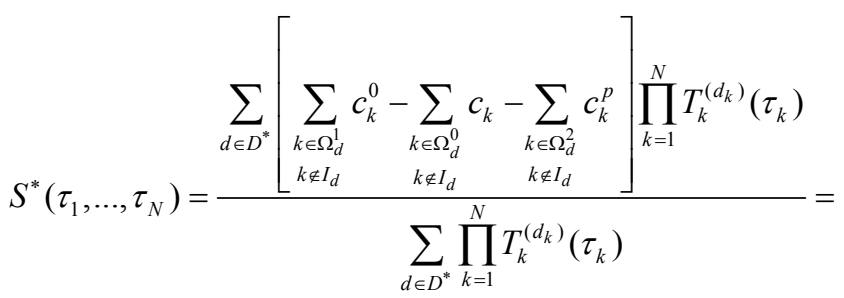

$$
\begin{aligned}
& \left\{\sum_{i=1}^{\infty}\left[\sum_{\substack{j \in M_{i} \\
j \notin A\left(M_{i}\right)}} c_{j}^{0} \prod_{n \in M_{i}} K_{n}\left(\tau_{n}\right) \prod_{\substack{n=1 \\
n \notin M_{i}}}^{N}\left(1-K_{n}\left(\tau_{n}\right)\right)-\sum_{j \notin M_{i}} C_{j}\left(\tau_{j}\right) K_{j}\left(\tau_{j}\right) \prod_{n \in M_{i}} K_{n}\left(\tau_{n}\right) \prod_{\substack{n \notin M_{i} \\
n \neq j}}\left(1-K_{n}\left(\tau_{n}\right)\right)\right]-\right. \\
& \left.\sum_{i=1}^{s} \sum_{\substack{j \in \Phi_{i} \\
j \notin A\left(\Phi_{i}\right)}} C_{j}\left(\tau_{j}\right) K_{j}\left(\tau_{j}\right) \prod_{\substack{n=1 \\
n \notin \Phi_{i}}}^{N} K_{n}\left(\tau_{n}\right) \prod_{\substack{n \in \Phi_{i} \\
n \neq j}}\left(1-K_{n}\left(\tau_{n}\right)\right)\right\} / \\
& {\left[\sum_{i=1}^{\infty} \prod_{n \in M_{i}} K_{n}\left(\tau_{n}\right) \prod_{\substack{n=1 \\
n \notin M_{i}}}^{N}\left(1-K_{n}\left(\tau_{n}\right)\right)+\sum_{i=1}^{s} \prod_{n=1}^{N} K_{n}\left(\tau_{n}\right) \prod_{n \in \Phi_{i}}\left(1-K_{n}\left(\tau_{n}\right)\right)\right]} \\
& C^{*}\left(\tau_{1}, \ldots, \tau_{N}\right)=\frac{\sum_{d \in D^{*}}\left[\sum_{\substack{k \in \Omega_{d}^{0} \\
k \notin I_{d}}} c_{k}+\sum_{\substack{k \in \Omega_{d}^{2} \\
k \notin I_{d}}} c_{k}^{p}\right] \prod_{k=1}^{N} T_{k}^{\left(d_{k}\right)}\left(\tau_{k}\right)}{\sum_{d \in D_{+}^{*}} \prod_{k=1}^{N} T_{k}^{\left(d_{k}\right)}\left(\tau_{k}\right)}=\left\{\sum_{i=1}^{\infty} \sum_{j \notin M_{i}} C_{j}\left(\tau_{j}\right) K_{j}\left(\tau_{j}\right) \prod_{n \in M_{i}} K_{n}\left(\tau_{n}\right) \prod_{\substack{n \notin M_{i} \\
n \neq j}}\left(1-K_{n}\left(\tau_{n}\right)\right)+\right. \\
& \left.\sum_{i=1}^{s} \sum_{\substack{j \in \Phi_{i} \\
j \notin A\left(\Phi_{i}\right)}} C_{j}\left(\tau_{j}\right) K_{j}\left(\tau_{j}\right) \prod_{\substack{n=1 \\
n \notin \Phi_{i}}}^{N} K_{n}\left(\tau_{n}\right) \prod_{\substack{n \in \Phi_{i} \\
n \neq j}}\left(1-K_{n}\left(\tau_{n}\right)\right)\right\} /\left[\sum_{i=1}^{\infty} \prod_{n \in M_{i}} K_{n}\left(\tau_{n}\right) \prod_{\substack{n=1 \\
n \notin M_{i}}}^{N}\left(1-K_{n}\left(\tau_{n}\right)\right)\right]
\end{aligned}
$$

Here $C_{i}\left(\tau_{i}\right)=\frac{c_{i}^{p} T_{i}^{(2)}\left(\tau_{i}\right)+c_{i} T_{i}^{(0)}\left(\tau_{i}\right)}{T_{i}^{(1)}\left(\tau_{i}\right)}$ are mean specific expenses per time unit of $i$-element's up state.

\section{Optimization of Elements' Maintenance Terms}

The task of defining optimal terms of elements' mainte- nance execution with the purpose of gaining the best system's performance index is reduced to the definition of the points of absolute extremum $\tau_{i}^{u}, \tau_{i}^{s}, \tau_{i}^{c}$ of the functions (10), (12) and (13) respectively. The attainment of function's extremums under some arguments $\tau_{j} \rightarrow \infty$ signifies that it is not expedient to execute maintenance of elements with respective numbers. In this case we should change $K_{j}(\infty)$ for $\frac{M \alpha_{j}}{M \alpha_{j}+M \beta_{j}}$, and $C_{j}(\infty)$ 
for $\frac{c_{j} M \beta_{j}}{M \alpha_{j}}$ in the Formulas (10), (12) and (13).

Let us write down formulas for the definition of stationary characteristics of multicomponent systems with concrete structures.

Stationary characteristics of serial system. The structure including $N$ elements in series has one path $M_{1}$ $=\{1, \ldots, N\}$ and $\mathrm{N}$ sections $\left\{\Phi_{i}\right\}_{i=1}^{N}=\{\{1\},\{2\}, \ldots,\{N\}\}$. System stationary performance indexes (8)-(10), (12) and (13) will be given by:

$$
\begin{gathered}
K_{u}^{*}\left(\tau_{1}, \ldots, \tau_{N}\right)=\left[1+\sum_{i=1}^{N} \frac{1-K_{i}\left(\tau_{i}\right)}{K_{i}\left(\tau_{i}\right)}\right]^{-1}, \\
S^{*}\left(\tau_{1}, \ldots, \tau_{N}\right)=\frac{\sum_{i=1}^{N} c_{i}^{0}-\sum_{i=1}^{N} C_{i}\left(\tau_{i}\right)}{1+\sum_{i=1}^{N} \frac{1-K_{i}\left(\tau_{i}\right)}{K_{i}\left(\tau_{i}\right)}}
\end{gathered}
$$

$$
\begin{gathered}
C^{*}\left(\tau_{1}, \ldots, \tau_{N}\right)=\sum_{i=1}^{N} C_{i}\left(\tau_{i}\right), \\
T_{+}^{*}\left(\tau_{1}, \ldots, \tau_{N}\right)=\frac{1}{\sum_{i=1}^{N} \frac{1}{T_{i}^{(1)}\left(\tau_{i}\right)},} \\
T_{-}^{*}\left(\tau_{1}, \ldots, \tau_{N}\right)=\frac{\sum_{i=1}^{N} \frac{T_{i}^{(0)}\left(\tau_{i}\right)+T_{i}^{(2)}\left(\tau_{i}\right)}{T_{i}^{(1)}\left(\tau_{i}\right)}}{\sum_{i=1}^{N} \frac{1}{T_{i}^{(1)}\left(\tau_{i}\right)}} .
\end{gathered}
$$

Stationary characteristics of parallel-serial system. The block scheme of the parallel-serial system is shown in Figure 2.

For the system of the structure like this the Formulas (10), (12) and (13) for the system stationary characteristics definition are as follows:

$$
\begin{aligned}
& K_{u}^{*}\left(\tau_{11}, \ldots, \tau_{L N_{L}}\right)=1-\prod_{i=1}^{L}\left(1-\left[1+\sum_{n=1}^{N_{i}} \frac{1-K_{\text {in }}\left(\tau_{\text {in }}\right)}{K_{\text {in }}\left(\tau_{\text {in }}\right)}\right]^{-1}\right), \\
& S^{*}\left(\tau_{11}, \ldots, \tau_{L N_{L}}\right)=\sum_{i=1}^{L} 1+\left[1+\sum_{n=1}^{N_{i}} \frac{1-K_{\text {in }}\left(\tau_{\text {in }}\right)}{K_{\text {in }}\left(\tau_{\text {in }}\right)}\right]^{-1} \sum_{n=1}^{N_{i}} \frac{S_{\text {in }}\left(\tau_{\text {in }}\right)}{K_{\text {in }}\left(\tau_{\text {in }}\right)}, \\
& C^{*}\left(\tau_{11}, \ldots, \tau_{L N_{L}}\right)=\frac{1}{K_{u}^{*}\left(\tau_{11}, \ldots, \tau_{L N_{L}}\right)} \sum_{i=1}^{L} \sum_{n=1}^{N_{i}} C_{i n}\left(\tau_{i n}\right)\left[1+\sum_{n=1}^{N_{i}} \frac{1-K_{\text {in }}\left(\tau_{\text {in }}\right)}{K_{\text {in }}\left(\tau_{\text {in }}\right)}\right]^{-1},
\end{aligned}
$$

where $K_{i n}\left(\tau_{i n}\right), S_{i n}\left(\tau_{i n}\right), C_{i n}\left(\tau_{i n}\right)$ are SSAF, mean specific income of $i$-chain's $n$-element per calendar time unit, and mean specific expenses per time unit of element's up state respectively:

$$
\begin{aligned}
& K_{\text {in }}\left(\tau_{\text {in }}\right)=\frac{T_{i n}^{(1)}\left(\tau_{\text {in }}\right)}{T_{\text {in }}^{(1)}\left(\tau_{\text {in }}\right)+T_{\text {in }}^{(0)}\left(\tau_{\text {in }}\right)+T_{\text {in }}^{(2)}\left(\tau_{\text {in }}\right)}, \\
& S_{i n}\left(\tau_{i n}\right)=\frac{c_{i n}^{0} T_{i n}^{(1)}\left(\tau_{i n}\right)-c_{i n} T_{i n}^{(0)}\left(\tau_{i n}\right)-c_{i n}^{p} T_{i n}^{(2)}\left(\tau_{\text {in }}\right)}{T_{i n}^{(1)}\left(\tau_{\text {in }}\right)+T_{\text {in }}^{(0)}\left(\tau_{\text {in }}\right)+T_{\text {in }}^{(2)}\left(\tau_{\text {in }}\right)}, \\
& C_{i n}\left(\tau_{i n}\right)=\frac{c_{i n} T_{i n}^{(0)}\left(\tau_{i n}\right)+c_{i n}^{p} T_{i n}^{(2)}\left(\tau_{i n}\right)}{T_{i n}^{(1)}\left(\tau_{i n}\right)}, \\
& T_{i n}^{(1)}\left(\tau_{i n}\right)=\int_{0}^{\tau_{i n}} \bar{F}_{i n}(s) d s, \\
& T_{i n}^{(2)}\left(\tau_{i n}\right)=M \beta_{i n}^{p} \bar{F}_{i n}\left(\tau_{i n}\right), \\
& T_{\text {in }}^{(0)}\left(\tau_{\text {in }}\right)=M \beta_{\text {in }} F_{\text {in }}\left(\tau_{\text {in }}\right) .
\end{aligned}
$$

Stationary characteristics of serial-parallel system.
The block scheme of serial-parallel system is shown in Figure 3.

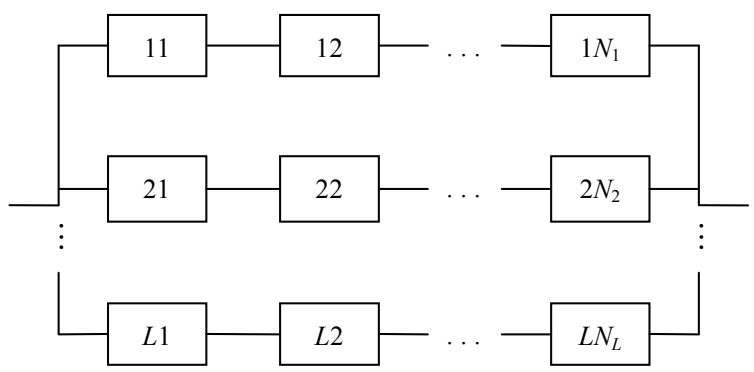

Figure 2. Block scheme of parallel-serial system.

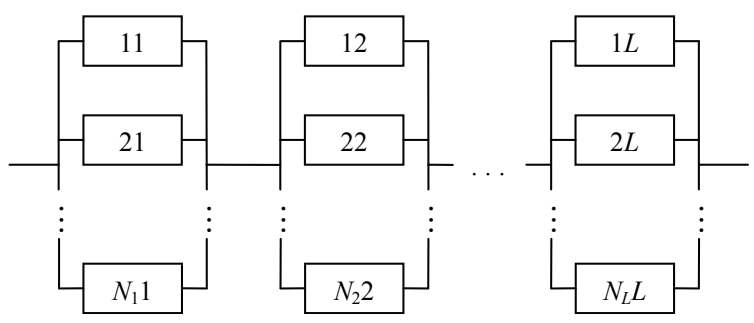

Figure 3. Block scheme of serial-parallel system. 
System stationary performance indexes are defined by the formulas:

$$
\begin{gathered}
K_{u}^{*}\left(\tau_{11}, \ldots, \tau_{L N_{L}}\right)=\left[1+\sum_{i=1}^{L} \frac{\prod_{n=1}^{N_{i}}\left(1-K_{n i}\left(\tau_{n i}\right)\right)}{1-\prod_{n=1}^{N_{i}}\left(1-K_{n i}\left(\tau_{n i}\right)\right)}\right]^{-1}, \\
S_{u}^{*}\left(\tau_{11}, \ldots, \tau_{L N_{L}}\right)=K_{u}^{*}\left(\tau_{11}, \ldots, \tau_{L N_{L}}\right) \sum_{i=1}^{L} \frac{\sum_{n=1}^{N_{i}} S_{n i}\left(\tau_{n i}\right)}{1-\prod_{n=1}^{N_{i}}\left(1-K_{n i}\left(\tau_{n i}\right)\right)}, \\
C_{u}^{*}\left(\tau_{11}, \ldots, \tau_{L N_{L}}\right)=\sum_{i=1}^{L} \frac{\sum_{n=1}^{N_{i}} C_{n i}\left(\tau_{n i}\right) K_{n i}\left(\tau_{n i}\right)}{1-\prod_{n=1}^{N_{i}}\left(1-K_{n i}\left(\tau_{n i}\right)\right)} .
\end{gathered}
$$

where $K_{n i}\left(\tau_{n i}\right), S_{n i}\left(\tau_{n i}\right), C_{n i}\left(\tau_{n i}\right)$ are SSAF, mean specific income of the $i$-chain's $n$-element per calendar time unit, and mean specific expenses per time unit of element's up state:

$$
\begin{gathered}
K_{n i}\left(\tau_{n i}\right)=\frac{T_{n i}^{(1)}\left(\tau_{n i}\right)}{T_{n i}^{(1)}\left(\tau_{n i}\right)+T_{n i}^{(0)}\left(\tau_{n i}\right)+T_{n i}^{(2)}\left(\tau_{n i}\right)}, \\
S_{n i}\left(\tau_{n i}\right)=\frac{c_{n i}^{0} T_{n i}^{(1)}\left(\tau_{n i}\right)-c_{n i} T_{n i}^{(0)}\left(\tau_{n i}\right)-c_{n i}^{p} T_{n i}^{(2)}\left(\tau_{n i}\right)}{T_{n i}^{(1)}\left(\tau_{n i}\right)+T_{n i}^{(0)}\left(\tau_{n i}\right)+T_{n i}^{(2)}\left(\tau_{n i}\right)}, \\
C_{n i}\left(\tau_{n i}\right)=\frac{c_{n i} T_{n i}^{(0)}\left(\tau_{n i}\right)+c_{n i}^{p} T_{n i}^{(2)}\left(\tau_{n i}\right)}{T_{n i}^{(1)}\left(\tau_{n i}\right)}, \\
T_{n i}^{(1)}\left(\tau_{n i}\right)=\int_{0}^{\tau_{n i}} \bar{F}_{n i}(s) d s,
\end{gathered}
$$

$$
\begin{aligned}
& T_{n i}^{(2)}\left(\tau_{n i}\right)=M \beta_{n i}^{p} \bar{F}_{n i}\left(\tau_{n i}\right), \\
& T_{n i}^{(0)}\left(\tau_{n i}\right)=M \beta_{n i} F_{n i}\left(\tau_{n i}\right) .
\end{aligned}
$$

Let us make concrete calculations to define optimal maintenance execution terms for three-component serial system. Let operating time to failure and restoration time are disposed according to Erlang with densities

$f_{i}(t)=\lambda_{i} \frac{\left(\lambda_{i} t\right)^{m_{i}-1}}{\left(m_{i}-1\right) !} e^{-\lambda_{i} t}, \quad g_{i}(t)=\mu_{i} \frac{\left(\mu_{i} t\right)^{m_{i}-1}}{\left(m_{i}-1\right) !} e^{-\mu_{i} t}$,

$i=1,2,3$. Initial data and calculation results are represented in the Tables $\mathbf{1}$ and $\mathbf{2}$.

In the Table $2 K_{u}^{\infty}, S^{\infty}, C^{\infty}$ denote system performance indexes in case if elements' maintenance is not carried out. If elements attain optimal time to failure, their maintenance execution increases these indexes for $4.5 \%, 6.8 \%$ and $38.3 \%$ respectively.

\section{Conclusions}

In the present paper semi-Markovian model of the multicomponent restorable system operation with regard to elements' deactivation and maintenance in age has been built. With the help of this model an explicit form of reliability and economical stationary performance indexes for the system with assumption of a general form of elements' time to failure and restoration time distributions has been defined. The system stationary characteristics found are explicitly dependent on the periodicity of its elements' maintenance execution. This fact allows solving the problems of the characteristics' improvement. In the limiting case (when the periodicity of elements'

Table 1. System initial data.

\begin{tabular}{ccccccccc}
\hline № & $\gamma_{i}$ & $\theta_{i}$ & $M \alpha_{i}, h$ & $M \beta_{i}, h$ & $M \beta_{i}^{p}, h$ & $c_{i}^{0}, c . u . / h$ & $c_{i}, c . u . / h$ & $c_{i}^{p}, c . u . / h$ \\
\hline 1 & 2 & 50 & 44.311 & 5 & 1 & 5 & 1 & 0.2 \\
2 & 3 & 15 & 13.395 & 3 & 1 & 7 & 3 & 2 \\
3 & 4 & 20 & 18.128 & 4 & 0.5 & 9 & 3 & 1 \\
\hline
\end{tabular}

Table 2. Calculation results.

\begin{tabular}{ccccccccccc}
\hline № & $\tau_{i}^{k}, h$ & $K_{u}^{\max }$ & $K_{u}^{\infty}$ & $\tau_{i}^{s}, h$ & $\begin{array}{c}S^{\max } \\
\text { c.u./h }\end{array}$ & $\begin{array}{c}S^{\infty} \\
\text { c.u./h }\end{array}$ & $\tau_{i}^{c}, h \quad \begin{array}{c}C^{\max } \\
\text { c.u./h }\end{array}$ c.u./h \\
\hline 1 & 25.533 & & 23.131 & & & 15.608 & \\
2 & 9.548 & 0.916 & 0.869 & 8.982 & 18.553 & 16.393 & 7.694 & 0.507 & 1.373 \\
3 & 9.354 & & & & & & & & \\
\hline
\end{tabular}


maintenance execution increases infinitely) the stationary characteristics defined in the present work take the form of the well-known expressions for the characteristics of restorable system in case of the passive strategy of maintenance (elements' maintenance is not carried out) $[8,9]$.

\section{References}

[1] C. Valdez-Flores and R. M. Feldman, "A Survey of Preventive Maintenance Models for Stochastically Deteriorating Single-Unit Systems," Naval Research Logistics, Vol. 36, No. 4, 1989, pp. 419-446.

[2] D. I. Cho and M. Parlar, "A Survey of Maintenance Models for Multi-Unit Systems," European Journal of Operational Research, Vol. 51, No. 2, 1991, pp. 1-23.

[3] R. Dekker and R. A. Wildeman, "A Review of MultiComponent Maintenance Models with Economic Dependence," Mathematical Methods of Operations Research, Vol. 45, No. 3, 1997, pp. 411-435.
[4] F. Beichelt and P. Franken, "Zuverlässigkeit und Instanphaltung," Mathematische Methoden, VEB Verlag Technik, Berlin, 1983.

[5] R. E. Barlow and F. Proschan, "Mathematical Theory of Reliability," John Wiley and Sons, New York, 1965.

[6] V. A. Kashtanov and A. I. Medvedev, "The Theory of Complex Systems' Reliability (Theory and Practice)," European Center for Quality, Moscow, 2002.

[7] A. I. Peschansky, "Monotonous System Maintenance with Regard to Operating Time to Failure of Each Element," Industrial Processes Optimization, Vol. 11, 2009, pp. 77-83.

[8] V. S. Korolyuk and A. F. Turbin, "Markovian Restoration Processes in the Problems of System Reliability," Naukova dumka, Kiev, 1982.

[9] A. N. Korlat, V. N. Kuznetsov, M. I. Novikov and A. F. Turbin, "Semi-Markovian Models of Restorable and Service Systems," Shtiintsa, Kishinev, 1991.

[10] V. M. Shurenkov, "Ergodic Markovian Processes," Nauka, Moscow, 1989. 